\title{
BREAKING WAVES FOR THE PERIODIC TWO-COMPONENT CAMASSA-HOLM SYSTEM
}

\author{
FEI GUO
}

(Communicated by James E. Colliander)

ABSTRACT. We give a blow-up result for the periodic two-component CamassaHolm system modeling shallow water waves moving over a linear shear flow.

\section{INTRODUCTION}

In this paper, we study the following coupled two-component Camassa-Holm shallow water system on the unit circle $\mathbb{S}=\mathbb{R} / \mathbb{Z}$ :

$$
\left\{\begin{array}{l}
m_{t}-A u_{x}+2 m u_{x}+u m_{x}+\rho \rho_{x}=0, \quad t>0, x \in \mathbb{R}, \\
\rho_{t}+(\rho u)_{x}=0, \quad t>0, x \in \mathbb{R}, \\
u(0, x)=u_{0}(x), \rho(0, x)=\rho_{0}(x), \quad x \in \mathbb{R}, \\
u(t, x+1)=u(t, x), \rho(t, x+1)=\rho(t, x), \quad t \geq 0, x \in \mathbb{R},
\end{array}\right.
$$

where the variable $u(t, x)$ represents the horizontal velocity of the fluid, $m=u-u_{x x}$, $\rho(t, x)$ is related to the free surface elevation from equilibrium (or scalar density), and the parameter $A>0$ characterizes a linear underlying shear flow propagating in the positive direction of the $x$-coordinate (or the critical shallow water speed). All of those are measured in dimensionless units.

System (1.1) was first derived in [18. Constantin and Ivanov 11] derived it in the context of shallow water theory. Recently, Ivanov [16] gave a rigorous justification of the derivation of system (1.1) which is a valid approximation to the governing equations for water waves in the shallow water regime with nonzero constant vorticity.

System (1.1) is completely integrable [16, as it can be written as a compatibility condition of two linear systems (Lax pair) with a spectral parameter $\zeta$; that is,

$$
\begin{gathered}
\Psi_{x x}=\left[-\zeta^{2} \rho^{2}+\zeta\left(m-\frac{A}{2}\right)+\frac{1}{4}\right] \Psi, \\
\Psi_{t}=\left(\frac{1}{2 \zeta}-u\right) \Psi_{x}+\frac{1}{2} u_{x} \Psi
\end{gathered}
$$

Received by the editors September 13, 2011 and, in revised form, July 17, 2012.

2010 Mathematics Subject Classification. Primary 35B10, 35B44, 35G55, 35L05, 35Q35.

Key words and phrases. Two-component Camassa-Holm system, wave-breaking, blow-up, integrable.

This work was partially supported by the NNSF (11071141, 11271192) of China, "333" and Qing Lan Project of Jiangsu Province, the Natural Science Foundation of Jiangsu Province (BK2011777), and the NSF of the Jiangsu Higher Education Committee of China (11KJA110001). 
Moreover, this system admits the following conservation law:

$$
E=\int_{\mathbb{S}}\left(u^{2}+u_{x}^{2}+\rho^{2}\right) d x .
$$

Obviously, if $\rho=0$, then system (1.1) is reduced to the well-known CamassaHolm equation [1,2]

$$
u_{t}-A u_{x}-u_{t x x}+3 u u_{x}=2 u_{x} u_{x x}+u u_{x x x}, \quad x \in \mathbb{R}, t>0 .
$$

This equation is a model describing unidirectional propagation of surface waves on a shallow layer of water which is at rest at infinity 1,2. A rigorous justification of the derivation of the Camassa-Holm equation as an approach to the governing equations for water waves was recently provided by Constantin and Lannes [12. Equation (1.3) was also found independently as a model for nonlinear waves in cylindrical hyperelastic rods [13. Recently, it was claimed in [17] that the equation might be relevant to the modeling of tsunami; also see the discussion in [10].

The Camassa-Holm equation has many very interesting properties: soliton interaction, existence of both permanent waves and breaking waves, as well as integrability in the sense of an infinite-dimensional Hamiltonian system [4 9]. Therefore, the Camassa-Holm equation has the potential to become the new master equation for shallow water wave theory.

Many recent works are devoted to studying system (1.1); for instance see [3, 14, 15] and the references therein. As we know, the only way that a classical solution of system (1.1) may fail to exist for all time is that the wave may break. This means that the solution remains bounded while its slopes become unbounded at a finite time - this blow-up phenomena is referred to as wave-breaking.

In this paper we present a new blow-up result (Theorem 3.1) for system (1.1). For some initial data $\left(u_{0}, \rho_{0}\right)$, if $u_{0}$ are "asymmetric enough" and "small", while $\rho_{0}$ equals zero on the extremal points of $u_{0, x}(x)$, it is shown that the corresponding solution blows up in finite time (see Remark 3.2).

Throughout the paper, we identify all spaces of periodic functions with function spaces over $\mathbb{S}$, and for simplicity we drop $\mathbb{S}$ in our notation.

\section{Preliminaries}

In terms of $u$ and $\rho$, we obtain the equivalent form of system (1.1),

$$
\left\{\begin{array}{l}
u_{t}-u_{t x x}-A u_{x}++3 u u_{x}-2 u_{x} u_{x x}-u u_{x x x}+\rho \rho_{x}=0, \\
\quad t>0, x \in \mathbb{R}, \\
\rho_{t}+(\rho u)_{x}=0, \quad t>0, x \in \mathbb{R}, \\
u(0, x)=u_{0}(x), \rho(0, x)=\rho_{0}(x), \quad x \in \mathbb{R}, \\
u(t, x+1)=u(t, x), \rho(t, x+1)=\rho(t, x), \quad t \geq 0, x \in \mathbb{R} .
\end{array}\right.
$$

Note the operator $\left(1-\partial_{x}^{2}\right)^{-1}$ acting on $L^{2}$ has the representation

$$
\left[\left(1-\partial_{x}^{2}\right)^{-1} f\right](x)=\int_{\mathbb{S}} G(x-\xi) f(\xi) d \xi, f \in L^{2}(\mathbb{S}),
$$

with the Green's function

$$
G(x)=\frac{\cosh \left(x-[x]-\frac{1}{2}\right)}{2 \sinh \left(\frac{1}{2}\right)}, x \in \mathbb{S} .
$$


Here [.] stands for the integer part. Therefore, we can rewrite system (2.1) as the following quasi-linear evolution equation of hyperbolic type:

$$
\left\{\begin{array}{l}
u_{t}+u u_{x}=A G * u_{x}-\partial_{x} G *\left(u^{2}+\frac{1}{2} u_{x}^{2}+\frac{1}{2} \rho^{2}\right), \quad t>0, x \in \mathbb{R}, \\
\rho_{t}+(\rho u)_{x}=0, \quad t>0, x \in \mathbb{R} \\
u(0, x)=u_{0}(x), \rho(0, x)=\rho_{0}(x), \quad x \in \mathbb{R} \\
u(t, x+1)=u(t, x), \rho(t, x+1)=\rho(t, x), \quad t \geq 0, x \in \mathbb{R} .
\end{array}\right.
$$

We recall the local existence theory for the initial-value problem (2.3).

Theorem 2.1 ([3]). Given an initial data $\left(u_{0}, \rho_{0}\right) \in H^{s} \times H^{s-1}, s>\frac{3}{2}$, there exists a maximal $T=T\left(A,\left\|\left(u_{0}, \rho_{0}\right)\right\|_{H^{s} \times H^{s-1}}\right)>0$ and a unique solution

$$
(u, \rho) \in C\left([0, T) ; H^{s} \times H^{s-1}\right) \cap C^{1}\left([0, T) ; H^{s-1} \times H^{s-2}\right)
$$

of system (2.3). Moreover, the solution $(u, \rho)$ depends continuously on the initial value $\left(u_{0}, \rho_{0}\right)$ and the maximal time of existence $T>0$ is independent of $s$.

We will make use of the following series of lemmas in the proof of Theorem 3.1.

Lemma $2.2([6])$. Let $T>0$ and $v \in C^{1}\left([0, T) ; H^{2}\right)$. Then for every $t \in[0, T)$, there exists at least one point $\xi(t) \in \mathbb{S}$ with

$$
m(t):=\inf _{x \in \mathbb{S}}\left[v_{x}(t, x)\right]=v_{x}(t, \xi(t)) .
$$

The function $m(t)$ is absolutely continuous on $(0, T)$ with

$$
\frac{d m}{d t}=v_{t x}(t, \xi(t)) \quad \text { a.e. on }(0, T) .
$$

The next two lemmas concern the best constants for some Sobolev-type inequalities.

Lemma 2.3 ([20]). For all $f \in H^{1}$, the following inequality holds:

$$
G *\left(f^{2}+\frac{1}{2} f_{x}^{2}\right) \geq C_{0} f^{2}(x)
$$

with

$$
C_{0}=\frac{1}{2}+\frac{\arctan \left(\sinh \left(\frac{1}{2}\right)\right)}{2 \sinh \left(\frac{1}{2}\right)+2 \arctan \left(\sinh \left(\frac{1}{2}\right)\right) \sinh ^{2}\left(\frac{1}{2}\right)} \approx 0.869 .
$$

Moreover, $C_{0}$ is the optimal constant obtained by the function

$$
f_{0}=\frac{1+\arctan \left(\sinh \left(x-[x]-\frac{1}{2}\right)\right) \sinh \left(x-[x]-\frac{1}{2}\right)}{1+\arctan \left(\sinh \left(\frac{1}{2}\right)\right) \sinh \left(\frac{1}{2}\right)} .
$$

Lemma 2.4 ([19]). For every $f \in H^{1}$, we have

$$
\max _{x \in[0,1]} f^{2}(x) \leq \frac{e+1}{2(e-1)}\|f\|_{H^{1}}^{2},
$$

where the constant $\frac{e+1}{2(e-1)}$ is sharp, obtained by the function $f(\cdot)=G(x-\cdot)$. 
The next lemma is the mean-value theorem for Riemann-Stieltjes integrals.

Lemma $2.5([6])$. Let $g$ be a monotone function on $[a, b]$. Further, let $f$ be a real continuous function on $[a, b]$. Then there exists $a \xi \in[a, b]$ such that

$$
\int_{a}^{b} f(s) g(s) d s=g(a) \int_{a}^{\xi} f(s) d s+g(b) \int_{\xi}^{b} f(s) d s .
$$

\section{MAin RESUlt}

In this section, we will give and prove the main result of this paper.

The associated Lagrangian coordinates of system (2.3) are established by the initial-value problem

$$
\left\{\begin{array}{l}
\frac{\partial q}{\partial t}=u(t, q), \quad 0<t<T \\
q(0, x)=x, \quad x \in \mathbb{R}
\end{array}\right.
$$

where $u \in C^{1}\left([0, T], H^{s-1}\right)$ is the first component of the solution $(u, \rho)$ to system (2.3) with initial data $\left(u_{0}, \rho_{0}\right) \in H^{s} \times H^{s-1}\left(s>\frac{3}{2}\right)$, and $T>0$ is the maximal time of existence. By a direct calculation, we have

$$
q_{t x}(t, x)=u_{x}(t, q(t, x)) q_{x}(t, x) .
$$

Then,

$$
\frac{\partial q}{\partial x}=e^{\int_{0}^{t} u_{x}(\tau, q(\tau, x)) d \tau}>0, \quad \text { for } t>0, x \in \mathbb{R}
$$

which means that $q(t, \cdot): \mathbb{R} \rightarrow \mathbb{R}$ is a diffeomorphism of the line for every $t \in[0, T)$.

We are in the position to give the main result of this paper. Consider, as long as the solution $(u, \rho)$ to system $(2.3)$ is defined,

$$
m_{1}(t)=\min _{x \in \mathbb{S}}\left[u_{x}(t, x)\right], m_{2}(t)=\max _{x \in \mathbb{S}}\left[u_{x}(t, x)\right] .
$$

Theorem 3.1. Let $\left(u_{0}, \rho_{0}\right) \in H^{s} \times H^{s-1}\left(s>\frac{3}{2}\right)$ and $T>0$ be the maximal time of existence of the corresponding solution $(u, \rho)$ to system (2.3). Assume there exist $x_{1}, x_{2} \in \mathbb{S}$ such that

$$
\begin{aligned}
& u_{0, x}\left(x_{1}\right)=\inf _{x \in \mathbb{S}} u_{0, x}(x), \rho_{0}\left(x_{1}\right)=0, \\
& u_{0, x}\left(x_{2}\right)=\sup _{x \in \mathbb{S}} u_{0, x}(x), \rho_{0}\left(x_{2}\right)=0 .
\end{aligned}
$$

Assume further that

$$
m_{1}(0)+m_{2}(0) \leq-16 A-4 C_{1},
$$

where

$$
C_{1}=\sqrt{\left(1-C_{0}\right) \frac{e+1}{2(e-1)}} E,
$$

$E, C_{0}, m_{1}(t)$ and $m_{2}(t)$ are defined by (1.2), (2.6) and (3.2), respectively. Then the solution $(u, \rho)$ of system (2.3) blows up in finite time and the maximal existence time can be estimated as

$$
0<T<\frac{4}{8 A+2 C_{1}} .
$$


Proof. By Theorem 2.1 and a simple density argument, it is enough to prove this theorem for $s \geq 3$.

By Lemma 2.2, we can define $\xi(t) \in \mathbb{S}$ as

$$
m_{1}(t)=u_{x}(t, \xi(t))=\inf _{x \in \mathbb{S}} u_{x}(t, x), t \in[0, T) .
$$

Obviously,

$$
u_{x x}(t, \xi(t))=0, \quad \text { for a.e. } t \in[0, T) .
$$

Since $q(t, \cdot)$ defined by $(3.1)$ is a diffeomorphism of $\mathbb{R}$ for any $t \in[0, T)$, we see that there exists an $x_{1}(t) \in \mathbb{R}$ such that

$$
q\left(t, x_{1}(t)\right)=\xi(t), t \in[0, T) .
$$

(3.3) and (3.7) imply that

$$
m_{1}(0)=u_{x}(t, \xi(0))=\inf _{x \in \mathbb{S}} u_{0, x}(x)=u_{0, x}\left(x_{1}\right) ;
$$

therefore we can choose $\xi(0)=x_{1}$ and again by (3.3),

$$
\rho_{0}(\xi(0))=\rho_{0}\left(x_{1}\right)=0 .
$$

Along the trajectory $q(t, x)$ defined by $(3.1)$, the second equation of $(2.3)$ becomes

$$
\frac{d \rho(t, q(t, x))}{d t}=-u(t, q(t, x)) \rho(t, q(t, x)) .
$$

Set $x=x_{1}(t)$; integrating (3.12) and by (3.11), we have

$$
\rho\left(t, q\left(t, x_{1}(t)\right)\right)=\rho(t, \xi(t))=0, \forall t \in[0, T) .
$$

On the other hand, since

$$
\sup _{x \in \mathbb{S}}\left[u_{x}(t, x)\right]=-\inf _{x \in \mathbb{S}}\left[-u_{x}(t, x)\right],
$$

we can similarly define

$$
m_{2}(t)=u_{x}(t, \eta(t))=\sup _{x \in \mathbb{S}} u_{x}(t, x), t \in[0, T),
$$

so that

$$
u_{x x}(t, \eta(t))=0, \quad \text { for a.e. } t \in[0, T) .
$$

Moreover, there exists an $x_{2}(t) \in \mathbb{R}$ such that

$$
q\left(t, x_{2}(t)\right)=\eta(t), t \in[0, T),
$$

and

$$
\rho\left(t, q\left(t, x_{2}(t)\right)\right)=\rho(t, \eta(t))=0, \forall t \in[0, T) .
$$

Differentiating the first equation in (2.3) with respect to $x$ yields

$$
u_{t x}+u u_{x x}+\frac{1}{2} u_{x}^{2}-A G * u_{x x}=u^{2}+\frac{1}{2} \rho^{2}-G *\left(u^{2}+\frac{1}{2} u_{x}^{2}+\frac{1}{2} \rho^{2}\right),
$$


taking into account the fact $-\partial_{x}^{2} G * f=f-G * f$, for any $f \in L^{2}$. In view of the definitions of $m_{i}(t)(i=1,2)$ by $(3.2)$, setting $x=\xi(t)$ and $\eta(t)$ in $(3.17)$ respectively, we obtain for a.e. $t \in(0, T)$ that

$$
\begin{aligned}
& \frac{d m_{1}}{d t}+\frac{1}{2} m_{1}^{2}-A \int_{0}^{1} G(y) u_{x x}(t, \xi(t)-y) d y \\
= & u^{2}(t, \xi(t))-\int_{0}^{1} G(\xi(t)-y)\left(u^{2}(t, y)+\frac{1}{2} u_{x}^{2}(t, y)+\frac{1}{2} \rho^{2}(t, y)\right) d y
\end{aligned}
$$

and

$$
\begin{aligned}
& \frac{d m_{2}}{d t}+\frac{1}{2} m_{2}^{2}-A \int_{0}^{1} G(y) u_{x x}(t, \eta(t)-y) d y \\
= & u^{2}(t, \eta(t))-\int_{0}^{1} G(\eta(t)-y)\left(u^{2}(t, y)+\frac{1}{2} u_{x}^{2}(t, y)+\frac{1}{2} \rho^{2}(t, y)\right) d y,
\end{aligned}
$$

where use has been made of (3.8), (3.13) and (3.15)-(3.16).

Note that $G(x) \geq 0$, and thanks to $(2.5)$ and $(2.7)$, we infer that

$$
\begin{aligned}
& u^{2}(t, x)-\int_{0}^{1} G(x-y)\left(u^{2}(t, y)+\frac{1}{2} u_{x}^{2}(t, y)+\frac{1}{2} \rho^{2}(t, y)\right) d y \\
\leq & \left(1-C_{0}\right) \frac{e+1}{2(e-1)}\|u\|_{H^{1}}^{2} \leq\left(1-C_{0}\right) \frac{e+1}{2(e-1)} E^{2}:=C_{1}^{2} .
\end{aligned}
$$

The function $g(y)=G(y)-\frac{1}{2 \sinh \left(\frac{1}{2}\right)}, y \in \mathbb{R}$, is continuous, decreasing on $\left[0, \frac{1}{2}\right]$ and increasing on $\left[\frac{1}{2}, 1\right]$, with $g\left(\frac{1}{2}\right)=0, g(0)=g(1) \leq \frac{1}{2}$. Note that by the spatial periodicity of $u_{x x}$ we find for $i=1,2$ that

$$
\begin{aligned}
& \left|\int_{0}^{1} G(y) u_{x x}(x-y, t) d y\right|=\left|\int_{0}^{1} g(y) u_{x x}(x-y, t) d y\right| \\
\leq & \left|\int_{0}^{\frac{1}{2}} g(y) u_{x x}(x-y, t) d y\right|+\left|\int_{\frac{1}{2}}^{1} g(y) u_{x x}(x-y, t) d y\right| .
\end{aligned}
$$

Applying Lemma 2.5 to estimate each of the two integrals on the right-hand side, we find

$$
\left|\int_{0}^{1} G(y) u_{x x}(x-y, t) d y\right| \leq m_{2}(t)-m_{1}(t), t \in[0, T] .
$$

Substituting (3.20)-(3.21) into (3.18) and (3.19), respectively, we deduce for a.e. $t \in[0, T)$ that

$$
\frac{d m_{1}(t)}{d t} \leq-\frac{1}{2} m_{1}^{2}+A\left(m_{2}-m_{1}\right)+C_{1}^{2}
$$

and

$$
\frac{d m_{2}(t)}{d t} \leq-\frac{1}{2} m_{2}^{2}+A\left(m_{2}-m_{1}\right)+C_{1}^{2}
$$

Summing up the above two inequalities gives

$$
\begin{aligned}
\frac{d\left(m_{1}+m_{2}\right)}{d t} & \leq-\frac{1}{2} m_{1}^{2}-\frac{1}{2} m_{2}^{2}+2 A\left(m_{2}-m_{1}\right)+2 C_{1}^{2} \\
& =-\frac{1}{2} m_{1}^{2}-\frac{1}{2} m_{2}^{2}+2 A\left(m_{2}+m_{1}\right)-4 A m_{1}+2 C_{1}^{2} .
\end{aligned}
$$


Hence if $\left(m_{1}+m_{2}\right)(0) \leq-16 A-4 C_{1}$, it remains so for all $t \in(0, T)$. Indeed, since $\bar{m}(t)=:\left(m_{1}+m_{2}\right)(t)+16 A+4 C_{1}$ is continuous on $[0, T)$, if the above inequality does not hold, we can find a $t_{0} \in(0, T)$ such that $\bar{m}\left(t_{0}\right)>0$. Denote

$$
t_{1}=\max \left\{t<t_{0}: \bar{m}(t)=0\right\} .
$$

Then

$$
\bar{m}\left(t_{1}\right)=0 \quad \text { and } \bar{m}^{\prime}\left(t_{1}\right) \geq 0
$$

But, due to

$$
m_{1}\left(t_{1}\right) \leq \frac{1}{2}\left(m_{1}+m_{2}\right)\left(t_{1}\right)=-8 A-2 C_{1},
$$

we have by (3.24) that

$$
\begin{aligned}
\bar{m}^{\prime}\left(t_{1}\right) & =\left(m_{1}+m_{2}\right)^{\prime}\left(t_{1}\right) \\
& =-\frac{1}{2} m_{1}^{2}\left(t_{1}\right)-\frac{1}{2} m_{2}^{2}\left(t_{1}\right)+2 A\left(m_{2}+m_{1}\right)\left(t_{1}\right)-4 A m_{1}\left(t_{1}\right)+2 C_{1}^{2} \\
& \leq-\frac{1}{2} m_{1}^{2}\left(t_{1}\right)-4 A m_{1}\left(t_{1}\right)+2 C_{1}^{2} \\
& <0 .
\end{aligned}
$$

This yields a contradiction with (3.25).

Putting the obtained estimate $m_{1}(t) \leq-8 A-2 C_{1}$ back into (3.22), we find

$$
\begin{aligned}
\frac{d m_{1}(t)}{d t} & \leq-\frac{1}{2} m_{1}^{2}+A\left(m_{1}+m_{2}\right)-2 A m_{1}+C_{1}^{2} \\
& \leq-\frac{1}{2} m_{1}^{2}-2 A m_{1}+C_{1}^{2} \\
& <-\frac{1}{4} m_{1}^{2}, \text { for a.e. } t \in(0, T) .
\end{aligned}
$$

Since $m_{1}(0)<-8 A-2 C_{1}$ on $[0, T)$ and locally Lipshitz on $(0, T)$, we see that $\frac{1}{m_{1}(t)}$ is also Lipshitz on $(0, T)$. Being locally Lipshitz, $\frac{1}{m_{1}(t)}$ is absolutely continuous on $(0, T)$. It is then inferred from (3.26) that

$$
\frac{d}{d t}\left(\frac{1}{m_{1}(t)}\right)>\frac{1}{4}, \quad \text { for a.e. } t \in(0, T) \text {. }
$$

Consequently,

$$
m_{1}(t)<\frac{4 m_{1}(0)}{4+m_{1}(0) t}, \quad \text { for a.e. } t \in(0, T) .
$$

This implies that $T<\frac{-4}{m_{1}(0)}$ and completes the proof of Theorem 3.1.

Remark 3.2. The technique of the proof differs from the ones used in the limiting case (i.e. $A=0$ ): the method adopted (for instance Theorem 3.4 in [3] and Theorem 4.2 in [14]) is based on the observation that an antisymmetric initial data develops into a spatially antisymmetric solution (this is not true for $A \neq 0$ if we deal with the nontrivial solution).

Remark 3.3. If $\rho \equiv 0,(3.5)$ in Theorem 3.1 is relaxed compared with Theorem 4.3 in [9. In fact, if $\rho \equiv 0$, then (3.5) becomes

$$
m_{1}(0)+m_{2}(0) \leq-16 A-4 C_{1} \approx-16 A-1.688\left\|u_{0}\right\|_{H^{1}} .
$$


The corresponding restriction in Theorem 4.3 in 9 is

$$
m_{1}(0)+m_{2}(0) \leq-32 A-2 \sqrt{3}\left\|u_{0}\right\|_{H^{1}} .
$$

Obviously, $-16 A-1.688\left\|u_{0}\right\|_{H^{1}}>-32 A-2 \sqrt{3}\left\|u_{0}\right\|_{H^{1}}$; therefore the restriction of $m_{1}(0)$ and $m_{2}(0)$ is relaxed.

\section{REFERENCES}

[1] Roberto Camassa and Darryl D. Holm, An integrable shallow water equation with peaked solitons, Phys. Rev. Lett. 71 (1993), no. 11, 1661-1664, DOI 10.1103/PhysRevLett.71.1661. MR.1234453 (94f:35121)

[2] R. Camassa, D. D. Holm and J. Hyman, A new integrable shallow water equation, Adv. in Appl. Mech. 31 (1994), 1-33.

[3] C. X. Chen and S. Wen, Wave breaking phenomena and global solutions for a generalized periodic two-component Camassa-Holm system, submitted.

[4] Adrian Constantin, Existence of permanent and breaking waves for a shallow water equation: a geometric approach (English, with English and French summaries), Ann. Inst. Fourier (Grenoble) 50 (2000), no. 2, 321-362. MR1775353 (2002d:37125)

[5] A. Constantin, On the blow-up of solutions of a periodic shallow water equation, J. Nonlinear Sci. 10 (2000), no. 3, 391-399, DOI 10.1007/s003329910017. MR:1752603 (2001b:35257)

[6] Adrian Constantin and Joachim Escher, Wave breaking for nonlinear nonlocal shallow water equations, Acta Math. 181 (1998), no. 2, 229-243, DOI 10.1007/BF02392586. MR.1668586 (2000b:35206)

[7] Adrian Constantin and Joachim Escher, Well-posedness, global existence, and blowup phenomena for a periodic quasi-linear hyperbolic equation, Comm. Pure Appl. Math. 51 (1998), no. 5, 475-504, DOI 10.1002/(SICI)1097-0312(199805)51:5〈475::AID-CPA2 $\rangle 3.0 . C O$; 2-5. MR 1604278 (98k:35165)

[8] Adrian Constantin and Joachim Escher, On the blow-up rate and the blow-up set of breaking waves for a shallow water equation, Math. Z. 233 (2000), no. 1, 75-91, DOI 10.1007/PL00004793. MR.1738352 (2001b:35258)

[9] Adrian Constantin and Joachim Escher, On the structure of a family of quasilinear equations arising in shallow water theory, Math. Ann. 312 (1998), no. 3, 403-416, DOI 10.1007/s002080050228. MR1654808 (2000b:35249)

[10] A. Constantin and R. S. Johnson, Propagation of very long water waves, with vorticity, over variable depth, with applications to tsunamis, Fluid Dynam. Res. 40 (2008), no. 3, 175-211, DOI 10.1016/j.fluiddyn.2007.06.004. MR2369543 (2009b:76009)

[11] Adrian Constantin and Rossen I. Ivanov, On an integrable two-component CamassaHolm shallow water system, Phys. Lett. A $\mathbf{3 7 2}$ (2008), no. 48, 7129-7132, DOI 10.1016/j.physleta.2008.10.050. MR2474608 (2009m:35418)

[12] Adrian Constantin and David Lannes, The hydrodynamical relevance of the Camassa-Holm and Degasperis-Procesi equations, Arch. Ration. Mech. Anal. 192 (2009), no. 1, 165-186, DOI 10.1007/s00205-008-0128-2. MR2481064 (2010f:35334)

[13] H.-H. Dai, Model equations for nonlinear dispersive waves in a compressible Mooney-Rivlin rod, Acta Mech. 127 (1998), no. 1-4, 193-207, DOI 10.1007/BF01170373. MR1606738 (98m:73037)

[14] Joachim Escher, Olaf Lechtenfeld, and Zhaoyang Yin, Well-posedness and blow-up phenomena for the 2-component Camassa-Holm equation, Discrete Contin. Dyn. Syst. 19 (2007), no. 3, 493-513, DOI 10.3934/dcds.2007.19.493. MR2335761 (2008j:35154)

[15] Chunxia Guan and Zhaoyang Yin, Global weak solutions for a two-component CamassaHolm shallow water system, J. Funct. Anal. 260 (2011), no. 4, 1132-1154, DOI 10.1016/j.jfa.2010.11.015. MR2747018(2011m:35238)

[16] Rossen Ivanov, Two-component integrable systems modelling shallow water waves: the constant vorticity case, Wave Motion 46 (2009), no. 6, 389-396, DOI 10.1016/j.wavemoti.2009.06.012. MR2598636(2011a:35415)

[17] M. Lakshmanan, Integrable nonlinear wave equations and possible connections to tsunami dynamics, Tsunami and nonlinear waves, Springer, Berlin, 2007, pp. 31-49, DOI 10.1007/9783-540-71256-5_2. MR2364923 (2008m:35309) 
[18] Peter J. Olver and Philip Rosenau, Tri-Hamiltonian duality between solitons and solitarywave solutions having compact support, Phys. Rev. E (3) 53 (1996), no. 2, 1900-1906, DOI 10.1103/PhysRevE.53.1900. MR.1401317 (97c:35172)

[19] Zhaoyang Yin, On the blow-up of solutions of the periodic Camassa-Holm equation, Dyn. Contin. Discrete Impuls. Syst. Ser. A Math. Anal. 12 (2005), no. 3-4, 375-381. MR2127025

[20] Yong Zhou, Blow-up of solutions to a nonlinear dispersive rod equation, Calc. Var. Partial Differential Equations 25 (2006), no. 1, 63-77, DOI 10.1007/s00526-005-0358-1. MR2183855 (2006k:35286)

School of Mathematical Sciences and Jiangsu Key Laboratory for NSLSCS, Nanjing Normal University, Nanjing 210046, People's Republic of China

E-mail address: guof@njnu.edu.cn

E-mail address: fgao@uta.edu 